\title{
Comparison of bronchial brushing and sputum in detection of pediatric pulmonary tuberculosis
}

\author{
Qiao-pei Chen', Shi-feng Ren ${ }^{2}$, Xin-feng Wang ${ }^{3^{*}}$ and Mao-shui Wang ${ }^{3^{*}}$
}

\begin{abstract}
The retrospective study aimed to evaluate the diagnostic value of bronchial brushing and sputum using acid fast bacilli smear, mycobacterial culture and real-time PCR in detection of pediatric pulmonary tuberculosis, sensitivity and specificity of bronchial brushing and sputum examined by the three methods were calculated and compared to each other. Data showed there were no significant difference in sensitivity between bronchial brushing and matched sputum using each method. But the specificity of real-time PCR on bronchial brushing was lower than on sputum. Compared with bronchial brushing, sputum was better specimen in detection of pediatric pulmonary tuberculosis.
\end{abstract}

Keywords: Bronchial brushing, Sputum, Pediatric pulmonary tuberculosis, Diagnosis

\section{Dear editor,}

Pediatric tuberculosis (TB) is often not considered a priority by national TB control programs, because the paucibacillary nature makes limited contribution to TB transmission. WHO estimated that in 2012 there were 530000 TB cases among children and 74 000 deaths among HIV-negative children [1]. Diagnosing pediatric $\mathrm{TB}$ is challenging, because of non-specific symptoms, difficulties in obtaining samples for microbiological examination and paucibacillary nature of their disease. Bronchial brushing is commonly used in human medicine as a diagnostic tool to sample neoplastic lesions or for microbiological analysis [2]. In the retrospective study, the role of bronchial brushing for diagnosing pediatric pulmonary TB was evaluated while compared with of sputum.

\section{Materials and methods}

Between Jul, 2007 and Apr, 2015, bronchial brushing via the flexible fiberoptic bronchoscope (Erbokryo CA, Erbe, Germany) and matched sputum were examined by acid fast bacilli (AFB) smear (Auramine O stain), mycobacterial

\footnotetext{
* Correspondence: 13789821006@163.com; wangmaoshui@gmail.com ${ }^{3}$ Department of Lab Medicine, Shandong Provincial Chest Hospital, 46\# Lishan Road, Jinan 250013Shandong, PR China

Full list of author information is available at the end of the article
}

culture (Lowenstein Jensen medium) and real-time PCR (Polymerase chain reaction) (TB RT-PCR kit, DAAN, China) in 59 pediatric patients ( $<15$ years old). 8 patients were excluded for uncertain diseases. The reaming 51 patients ( $12.3 \pm 1.4$ years old, 26 male) with determined diseases were enrolled, and then were divided into pulmonary TB group (27 cases, $12.7 \pm 1.3$ years old, 12 male) and control group (24 cases, $11.8 \pm 1.4$ years old, 14 male). Pediatric pulmonary TB patients were diagnosed based on TB contact, clinical symptoms (fever, anorexia, weight loss, cough and a response to anti-tuberculosis therapy), chest X-ray examination, tuberculin skin test and routine TB assays. Patients were classified as control subjects when an alternative diagnosis was established. Differences in sensitivity and specificity between specimens were estimated by the McNemar's test, with $P<0.05$ considered significant. All calculations were estimated at a $95 \%$ confidence interval $(95 \% \mathrm{CI})$. The protocol was approved by the Ethical Committee of the institute. Written informed consent was waived because of the retrospective nature.

\section{Findings}

As shown in Table 1, the specificity (100\%, $95 \%$ CI: 86.2-100\%) of AFB smear, mycobacterial culture and combination of the three tests on bronchial brushing was equal (all $P>0.05)$ with on sputum $(100 \%, 95 \% \mathrm{CI}$ : 
Table 1 Performance of the both specimens using TB assays in detection of pediatric pulmonary TB

\begin{tabular}{|c|c|c|c|c|}
\hline & \multicolumn{2}{|l|}{ Sensitivity (95 \% CI) } & \multicolumn{2}{|l|}{ Specificity (95 \% Cl) } \\
\hline & Bronchial brushing & Sputum & Bronchial brushing & Sputum \\
\hline AFB smear & $29.6 \%(15.9-48.5 \%)$ & $33.3 \%$ (18.6-52.2 \%) & $100 \%$ (86.2-100 \%) & $100 \%(86.2-100 \%)$ \\
\hline Mycobacterial cutlrue & $37.0 \%(21.5-55.8 \%)$ & $44.4 \%$ (27.6-62.7 \%) & $100 \%$ (86.2-100 \%) & $100 \%(86.2-100 \%)$ \\
\hline Real-time PCR & $33.3 \%$ (18.6-52.2 \%) & 33.3 \% (18.6-52.2 \%) & 91.7 \% (74.2-97.7 \%) & $100 \%(86.2-100 \%)$ \\
\hline Combination & $48.2 \%(30.7-66.0 \%)$ & $55.6 \%$ (37.3-72.4 \%) & $100 \%$ (86.2-100 \%) & $100 \%(86.2-100 \%)$ \\
\hline
\end{tabular}

$C l$ confidence interval, $A F B$ acid-fast bacilli, $P C R$ polymerase chain reaction

86.2-100\%), but the specificity of real-time PCR (91.7 \%, 95 \% CI: 74.2-97.7\%) on bronchial brushing was lower than on sputum (100\%, 95 \% CI: 86.2-100 \%) $(P<$ $0.01)$. For detection of pediatric pulmonary TB, sensitivities of AFB smear, mycobacterial culture, real-time PCR, or combination of the three tests on both specimens were, (1) bronchial brushing, $29.6 \%$ (95 \% CI: 15.9-48.5\%), $37.0 \%$ (95 \% CI: 21.5-55.8 \%), $33.3 \%$ (95 \% CI: 18.6-52.2\%) and $48.2 \%$ (95\% CI: 30.7-66.0\%), respectively; (2)sputum, 33.3 \% (95 \% CI: 18.6-52.2 \%), $44.4 \%$ (95 \% CI: $27.6-62.7 \%), 33.3 \%$ (95 \% CI: 18.6-52.2 \%) and $55.6 \%$ (95\% CI: 37.3-72.4\%), respectively. McNemar's tests showed there were no significant difference in sensitivity between bronchial brushing and matched sputum using each method (all $P>0.05)$.

\section{Discussion}

Pediatric TB is difficult to diagnose, and efforts are made to improve its diagnostic accuracy, including trying different specimens, such as bronchoalveolar lavage fluid (BALF), induced sputum, nasopharynx swab [3]. Unfortunately, routine technologies performed on them cannot detect pediatric TB accurately [4]. Currently, few studies have been performed aiming to evaluate the diagnostic role of bronchial brushing in detection of pulmonary TB. Mehta J et al found that smears of brush/ wash specimens from fiberoptic bronchoscopy were of comparable sensitivity to those of sputum [5]. In our study, we showed the similar results. Meanwhile, mycobacterial culture and real-time PCR performed on bronchial brushing or sputum also have same sensitivities in detection of pediatric TB. We also compared BALF and sputum in detection of pediatric pulmonary TB (data not published). The three assays were all negative in BALF and sputum among Non-pulmonary TB patients (three cases); among the six pulmonary TB patients, three were BALF culture positive, one was real-time PCR positive in BALF, no one was positive in sputum using these assays. The results showed that the BALF tended to be better than sputum in detection of pediatric pulmonary TB in the help of the three assays. Since few cases (only three) were examined parallel in BALF and bronchial brushing using the three assays, further comparison of BALF, sputum and bronchial brushing weren't conducted. The limitations of the study included: (1) retrospective nature; (2) It was difficult to assess the utility of induced sputum and BALF, as they were performed in few children; (3) because of younger children usually have difficulty in producing a sputum, the enrolled subjects all aged $>10$ years old.

In conclusion, compared with bronchial brushing, sputum was better specimen in detection of pediatric pulmonary TB when using AFB smear, mycobacterial culture and real-time PCR.

\section{Abbreviations}

BALF: bronchoalveolar lavage fluid; PCR: polymerase chain reaction; TB: tuberculosis; 95 \% Cl: 95 \% confidence interval.

\section{Competing interests}

The authors declare that they have no competing interests.

\section{Authors' contributions}

WXF and WMS conceived and designed the study. CQP and RSF collected data. CQP and WMS have been involved in the analysis and interpretation of data. WMS and WXF wrote the manuscript. All authors read and approved the final manuscript.

\section{Acknowledgements}

This work was supported in part by a grant from the Health Department of Shandong Province (NO.2011HZ085); in part by a grant from the Science and Technology Department of Jinan (NO.201303043).

\section{Author details}

${ }^{1}$ Department of Clinical Laboratory, Guangxi Maternal and Child Health Hospital, Nanning, Guangxi, PR China. 'Department of Lab Medicine, Xingming Hospital, Lanling, Shandong, PR China. ${ }^{3}$ Department of Lab Medicine, Shandong Provincial Chest Hospital, 46\# Lishan Road, Jinan 250013Shandong, PR China.

Received: 10 September 2015 Accepted: 21 January 2016

Published online: 27 January 2016

\section{References}

1. Starke JR. Improving tuberculosis care for children in high-burden settings. Pediatrics. 2014;134:655-7.

2. Dionisio J. Diagnostic flexible bronchoscopy and accessory techniques. Rev Port Pneumol. 2012;18:99-106.

3. Wang X, Wu Y, Zhang K, Guan C, Gao X, Wang M. Value of real-time polymerase chain reaction in bronchoalveolar lavage fluid for diagnosis of pediatric pulmonary tuberculosis. Braz J Infect Dis. 2013;17:718-9.

4. Wang JL, Zhao GW, Zhang ZQ, Wang XF, Wang MS. Clinicopathologic characteristics of pediatric tuberculous pleural effusion: a retrospective analysis of 112 consecutive cases. Eur Rev Med Pharmacol Sci. 2015;19:2978-82.

5. Mehta J, Krish G, Berro E, Harvill L. Fiberoptic bronchoscopy in the diagnosis of pulmonary tuberculosis. South Med J. 1990;83:753-5. 